\title{
A time-reversal suboptimal detector for underwater acoustic barriers
}

\author{
S.M. Jesus and O.C. Rodríguez \\ ISR, University of Algarve \\ Campus de Gambelas, 8005-139 Faro \\ Portugal \\ Email: $\{$ sjesus,orodrig\} @ualg.pt
}

\begin{abstract}
This paper proposes an experimental setup composed of two interconnected vertical arrays: one transmit only array (TOA) and a receive only array (ROA). It is shown that using the time reversal principle, where the ocean is used as a spatial matched filter, signal energy can be simultaneously focused on each ROA hydrophone and thus obtain the detector output by simple summation of the received energy over the array. This setup effectively configures a multistatic system with several transmitters and several receivers coherently processed both in time and space. Simulations using a ray trace propagation model combined with a naive scattering formulation show that the obtained empirical detection probability is close to the theoretical optimal bound derived assuming space - time white Gaussian noise. This system was tested in a sea trial that took place during September 2007 in the Hopavågen Bay near Trondheim, Norway. The actual setup was composed of a 2 sources TOA on a shallow area of $8 \mathrm{~m}$ depth near the shore and a 16 receivers $\mathrm{ROA}$ approximately $100 \mathrm{~m}$ apart in a 8 to $25 \mathrm{~m}$ depth range dependent duct. The transmitted signals were $200 \mathrm{~ms}$ duration LFMs with $3 \mathrm{kHz}$ bandwidths in two frequency bands centered on 5 and $10 \mathrm{kHz}$. The results obtained in realistic conditions show that a $1.7 \mathrm{~m}^{2}$ hard plate could be detected when placed across the barrier. Several results are presented and compared with the theoretical values. Although the system can be significantely improved, in particular by using more populated source and receiving arrays, it is believed that these results can be reproduced at sea in harbor like conditions.
\end{abstract}

\section{INTRODUCTION}

The association between an high level of alert to terrorist attacks and the widespread of small autonomous underwater vehicles has prompted for the development of harbor protection systems. These systems, here referred to as underwater barriers, generally combine various techniques including magnetic sensors, mechanic steel nets and acoustic sensors. There are at least three main difficulties associated with the usage of acoustics in underwater barriers for harbor protection: very shallow water signal propagation and reverberation, strong interference from other ships and structures around the port and the requirement of a relatively high frequency for detecting small objects. As a rule of thumb the carrier frequency for underwater acoustic barriers target detection should be of the order of a fraction of the half wavelength of the target cross section. So, a cross section smaller than $0.5 \mathrm{~m}$ would lead to a carrier frequency higher than $6 \mathrm{kHz}$, in practice say between 5 and $15 \mathrm{kHz}$. Propagation prediction in very shallow water $(<30 \mathrm{~m} \mathrm{depth})$ and at those frequencies is extremely difficult with current propagation models that do not properly account for surface and bottom roughness and scattering.

Sonar target detection classically deals, in a NeymanPearson sense, with the comparison of the correlation between the received signal and the target echo with the correlation of the received signal with the non-target echo [1]. If the difference between those two quantities is larger than a given threshold (that depends on the allowed false alarm rate), the target is said to be present. The problem is that the received signal with the target echo is normally not available and thus the optimal detector can not be implemented. In practice, we are bound to suboptimal detectors that use estimates of the target/no-target signal estimates drawn from a pre-experiment or system training phase. In a monostatic configuration the normally used target echo is the backward scatter, while in a bistatic setup the target echo is the forward scatter that, alternatively, can be seen as the acoustic field perturbation produced by a target passing through (or close to) the barrier.

This paper presents a comparative study of several optimal and suboptimal detectors and respective implementations using a naive ray tracing forward scattering model in a realistic environment. This environment was similar to that encountered during the Underwater Acoustic Barriers 2007 (UAB07) experiment carried out in the Hopavågen Bay, Sletvik, $80 \mathrm{~km}$ west of Trondheim (Norway) during September 2007. The area was acoustically very quite, away from ship traffic or other noise sources or interferences. The sound speed profile was nearly isovelocity with only some variation at a few meters from the bottom. The bottom was composed of a rock sand layer of an unspecified thickness. The water surface was very calm with less than $0.25 \mathrm{~m}$ wave ripples. The experimental setup included a 2 emitters TOA and a 16 receivers ROA. Emitters and receivers were connected via a wireless link so as to allow for reciprocal transmission and several detector implementations. Unfortunately only 4 out of the 16 receivers could be used so a reduced performance on detecting a 1.7 $\mathrm{m}^{2}$ alluminium plate target simulator crossing the barrier was anticipated.

\section{THE STANDING FIELD DETECTOR}

\section{A. The optimal case}

A possible practical setup for implementing a target detector is formed by a standing field created between a TOA and a 
ROA. Normally this detection problem is tackled by a binary detector with the two candidate hypothesis

$$
\begin{aligned}
& H_{0} \quad \text { : there is no change in the signal } \\
& H_{1} \quad \text { : the signal has changed }
\end{aligned}
$$

where the objective is obviously to detect a change in the standing field. Let us assume that only one source is in action and that the respective signal is received in all $K$ ROA sensors and grouped in an augmented observation vector $\mathbf{y}_{a}$

$$
\begin{aligned}
\mathbf{y}_{a} & =\mathbf{H}_{a} \mathbf{s}+\mathbf{w}_{a} \\
& =\mathbf{x}_{a}+\mathbf{w}_{a},
\end{aligned}
$$

with

$$
\mathbf{y}_{a}^{t}=\left[\mathbf{y}^{t}(0), \mathbf{y}^{t}(1), \ldots, \mathbf{y}^{t}(N-1)\right],
$$

where $N$ is the number of time samples in a single snapshot and where the $K$-dimensional array vectors $\mathbf{y}(n)$ are defined as $\mathbf{y}^{t}(n)=\left[y_{1}(n), \ldots, y_{K}(n)\right]$, so that $\mathbf{y}_{a}$ is of dimension $K N \times 1$. The first term on the right hand side of (1) represents the convolution equation between the $K N \times M$ channel impulse response matrix $\mathbf{H}_{a}$ in a suitable format for the augmented observation vector and the $M$ dimensional source signal time vector $\mathbf{s}$, while $\mathbf{w}_{a}$ is the observation noise assumed zero mean, temporally and spatially white, independent from the signal and Gaussian distributed. Form (3) is the so-called temporal ordering. A similar notation could be used in a spatial ordering where the augmented vectors would be formed by $K$ vectors of $N$ dimensional time vectors.

The classical treatment points to the usage of the likelihood ratio test and the Neyman-Pearson criteria, where the received signal under the two candidate hypothesis are

$$
\begin{array}{ll}
H_{0} & : \quad \mathbf{y}_{a}=\mathbf{H}_{a} \mathbf{s}+\mathbf{w}_{a} \\
H_{1} & : \quad \mathbf{y}_{a}=\tilde{\mathbf{H}}_{a} \mathbf{s}+\mathbf{w}_{a},
\end{array}
$$

where $\tilde{\mathbf{H}}_{a}$ is the modified channel response matrix due to the target interference, giving rise to the modified noise-free signal $\tilde{\mathbf{x}}_{a}=\tilde{\mathbf{H}}_{a} \mathbf{s}$. In this case the optimal likelihood ratio detector using the time-ordering form is

$$
L_{t}\left(\mathbf{y}_{a}\right)=\sum_{n=0}^{N-1}\left[\mathbf{y}^{T}(n) \tilde{\mathbf{H}}(n) \mathbf{s}-\mathbf{y}^{T}(n) \mathbf{H}(n) \mathbf{s}\right] \geq \gamma,
$$

and for the spatial-ordering,

$$
L_{s}\left(\mathbf{y}_{a}\right)=\sum_{k=1}^{K}\left[\mathbf{y}_{k}^{T} \tilde{\mathbf{H}}_{k} \mathbf{s}-\mathbf{y}_{k}^{T} \mathbf{H}_{k} \mathbf{s}\right] \geq \gamma,
$$

where $\gamma$ is some given threshold. These are two equivalent expressions and for an economy of space we will present the results using only the spatial ordering notation. Expression (5) basically tells us that the optimal detector is given by the comparison of the correlation of the received signal $\mathbf{y}$ with the expected modified noise free signal $\tilde{\mathbf{H}}$ s and check if it is larger or equal than the correlation of the received signal with the expected unmodified noise free signal Hs plus some security (to avoid false alarms) threshold. If that is the case,
$H_{1}$ is in force and the target is assumed present, and said to be absent otherwise.

This classical result under the assumed hypothesis allows to derive the detector performance from the statistics

$$
L_{s}\left(\mathbf{y}_{a}\right): \begin{cases}\mathcal{N}\left(\epsilon_{x \tilde{x}}-\epsilon_{x}, \sigma^{2} \epsilon_{x-\tilde{x}}\right) & \text { under } H_{0} \\ \mathcal{N}\left(\epsilon_{\tilde{x}}-\epsilon_{x \tilde{x}}, \sigma^{2} \epsilon_{x-\tilde{x}}\right) & \text { under } H_{1}\end{cases}
$$

with the generic cross-energy expression

$$
\epsilon_{x \tilde{x}}=\sum_{n=0}^{N-1} \tilde{\mathbf{x}}^{T}(n) \mathbf{x}(n)
$$

and

$$
\epsilon_{x-\tilde{x}}=\sum_{n=0}^{N-1}[\underline{\mathbf{x}}(n)-\tilde{\mathbf{x}}(n)]^{T}[\mathbf{x}(n)-\tilde{\mathbf{x}}(n)] .
$$

Giving rise to the Receiver Operating Characteristics (ROC) expression

$$
P_{D}=Q\left[Q^{-1}\left(P_{F A}\right)-\sqrt{d^{2}}\right],
$$

where $Q$ is the tail distribution function of a standardized normal random distribution and $d^{2}=\epsilon_{x-\tilde{x}} / \sigma^{2}$.

In the case of multiple, say $J$, sound sources where the received signal can be written as a superposition of the $J$ source signals, it can be shown that similar detector expressions are obtained but where the unmodified received signal $\mathrm{x}$ and the modified received signal $\tilde{\mathbf{x}}$ are replaced respectively by

$$
\mathbf{z}(n)=\sum_{j=1}^{J} \mathbf{H}_{j}(n) \mathbf{s}_{j} \quad \text { and } \quad \tilde{\mathbf{z}}(n)=\sum_{j=1}^{J} \tilde{\mathbf{H}}_{j}(n) \mathbf{s}_{j},
$$

where $\mathbf{s}_{j}$ is the time waveform emitted by source $j$ and $\mathbf{H}_{j}$ (respectively $\tilde{\mathbf{H}}_{j}$ ) are the unmodified (respectively modified) $j$ th source channel response matrix. Therefore the ROC expression for the $J$ source case is given by (9) with $d^{2}=\epsilon_{z-\tilde{z}} / \sigma^{2}$.

\section{B. Suboptimal implementations}

The two detector expressions of the previous section were derived under optimal conditions and their performance effectively represent upper bounds for the practical implementation, which are normally considered, at best, as suboptimal. This section presents various practical possible implementations and derives their performance.

1) The trivial power-based detector: The trivial powerbased detector compares the received signal power from one ping to the next with a threshold increment. Therefore this implementation can be written as

$$
L_{s}\left(\mathbf{y}_{a}\right)=\sum_{k=1}^{K} \tilde{\mathbf{y}}_{k}^{T}[n+1] \tilde{\mathbf{y}}_{k}[n+1]-\mathbf{y}_{k}^{T}[n] \mathbf{y}_{k}[n] \geq \gamma^{\prime},
$$

under hypothesis $H_{1}$ while under hypothesis $H_{0}, \tilde{\mathbf{y}}_{k}[n+1]$ is obviously replaced by $\mathbf{y}_{k}[n+1]^{1}$. With the same noise distribution assumptions this detector is noncentral Chi-squared distributed. However, for a large number of terms on the summation this distribution tends to Gaussian and in particular

\footnotetext{
${ }^{1}(n)$ means true time while $[n]$ means snapshot time.
} 
as the cross-signal SNR $\ll$ the number of terms $2 N K$, its probability of detection can be approximated by

$$
P_{D} \approx Q\left[Q^{-1}\left(P_{F A}\right)-\frac{\epsilon_{\tilde{x}}-\epsilon_{x}}{2 \sigma^{2} \sqrt{N K}}\right] .
$$

2) The non-channel based detector: One of the problems in implementing the optimal expression (5) deals with the fact that the channel matrix $\mathbf{H}_{k}$ or, more importantly $\tilde{\mathbf{H}}_{k}$, are unknown. So, one of the possibilities is to consider that the underwater acoustic channel response is replaced by a simple Dirac impulse and therefore the received signal, under this hypothesis, would be equal to the emitted signal s.

The implementation calls for the same source signal $\mathbf{s}$ transmitted at all times and received, say at times $t_{n-1}$ and $t_{n}$, thus if the target enters the field at $t=t_{n}$,

$$
\begin{aligned}
t & =t_{n-1} \quad \mathbf{s} \rightarrow \mathbf{y}_{k}[n]=\mathbf{H}_{k} \mathbf{s}+\mathbf{w}_{k}[n] \\
t & =t_{n} \quad \mathbf{s} \rightarrow \tilde{\mathbf{y}}_{k}[n+1]=\tilde{\mathbf{H}}_{k} \mathbf{s}+\mathbf{w}_{k}[n+1]
\end{aligned}
$$

giving rise to the detector expressions

$$
L_{s}\left(\mathbf{y}_{a} / H_{1}\right)=\sum_{n=1}^{K} \tilde{\mathbf{y}}_{k}^{T}[n+1] \mathbf{s}-\mathbf{y}_{k}^{T}[n] \mathbf{s} \geq \gamma^{\prime},
$$

under hypothesis $H_{1}$ assuming a single source in spatially and temporally white noise. And under hypothesis $H_{0}$

$$
L_{s}\left(\mathbf{y}_{a} / H_{0}\right)=\sum_{k=1}^{K} \mathbf{y}^{T}[n+1] \mathbf{s}-\mathbf{y}^{T}[n] \mathbf{s} \geq \gamma^{\prime} .
$$

Thus the detector performance in terms of ROC is

$$
P_{D}=Q\left[Q^{-1}\left(P_{F A}\right)-\frac{\epsilon_{s \tilde{x}}-\epsilon_{s x}}{\sqrt{2 K \sigma^{2} \epsilon_{s}}}\right],
$$

where $\epsilon_{s}$ is the emitted signal energy.

3) The time-reversal double ping-based detector: This is the most sophisticated suboptimal detector implementation that approaches the optimal expression (5) using the timereversal (TR) principle of channel reciprocity and time invariance. In that case the signal received on the ROA is sent back to the transmit array by wire or wireless so it can be used for a reciprocal transmission into the same acoustic channel ${ }^{2}$. Let us assume that at time snapshot 0 there is no target crossing the barrier so the received signal is $\mathbf{y}_{k}[0]$. If an object gets through the barrier at time $t_{n-1}$ than the channel response will be (always for sensor $k$ ) $\tilde{\mathbf{y}}_{k}[n-1]$ if signal $\mathbf{s}$ is transmitted and $\tilde{\mathbf{q}}_{k}[n-1]$ if the time reversed signal $\mathbf{y}^{\prime}{ }_{k}[0]$ is transmitted ${ }^{3}$. So, under hypothesis $H_{1}$, at time $t_{n-1}$, the sequence of signals being transmitted are

$$
\begin{aligned}
& t=t_{n-1} \quad \mathbf{y}^{\prime}{ }_{k}[0] \rightarrow \tilde{\mathbf{q}}_{k}[n-1]=\tilde{\mathbf{H}}_{k} \mathbf{y}^{\prime}{ }_{k}[0]+\mathbf{w}_{k}[n-1] \\
& t=t_{n} \quad \mathbf{s} \rightarrow \tilde{\mathbf{y}}_{k}[n]=\tilde{\mathbf{H}}_{k} \mathbf{s}+\mathbf{w}_{k}[n] \\
& t=t_{n+1} \quad \tilde{\mathbf{y}}_{k}{ }_{k}[n] \rightarrow \tilde{\mathbf{q}}_{k}[n+1]=\tilde{\mathbf{H}}_{k} \tilde{\mathbf{y}}_{k}{ }_{k}[n]+\mathbf{w}_{k}[n+1]
\end{aligned}
$$

\footnotetext{
${ }^{2}$ this assumes the existence of a training phase during which the target is assumed not to be present and the sources alternatively transmit test signals one source at a time, as proposed in [2]

${ }^{3}$ prime' means time-reversal operation.
}

assuming that the target crosses the barrier at time $t=t_{n}$. The detector under $H_{1}$ becomes,

$$
\hat{L}_{s}^{T R 2}\left(\mathbf{y}_{a} / H_{1}\right)=\sum_{k=1}^{K} \mathbf{s}^{\prime T} \tilde{\mathbf{q}}_{k}[n+1]-\mathbf{s}^{\prime T} \tilde{\mathbf{q}}_{k}[n-1],
$$

and under $H_{0}$

$$
\hat{L}_{s}^{T R 2}\left(\mathbf{y}_{a} / H_{0}\right)=\sum_{k=1}^{K} \mathbf{s}^{\prime^{T}} \mathbf{q}_{k}[n+1]-\mathbf{s}^{\prime T} \mathbf{q}_{k}[n-1],
$$

which says that under $H_{0}$ the detector is the difference of two closely matched filter outputs while under $H_{1}$ the detector is the difference of a closely matched (the second term) and an unmatched filter output (the first term). So, in this case if the detector is to be compared to a positive threshold, the absolute value of the detector should be used or its sign reversed. From a practical point of view this requires a much simpler detector implementation that is simply based on the sum over receiver hydrophones of the matched-filter outputs at any time slot. As in the previous cases, a carefull study was carried out for the statistics of this detector taking into account the observation noise at the receiver on both transmissions. The distributions of $L_{s}$ under hypothesis $H_{0}$ and $H_{1}$ is

$$
L_{s}\left(\mathbf{y}_{a}\right): \begin{cases}\mathcal{N}\left[0,2 \sigma^{2}\left(K \epsilon_{s}+\epsilon_{x}\right)\right] & \text { under } H_{0} \\ \mathcal{N}\left[\epsilon_{\tilde{x}}-\epsilon_{x \tilde{x}}, 2 \sigma^{2}\left(\epsilon_{\tilde{x}}+K \epsilon_{s}\right)\right] & \text { under } H_{1}\end{cases}
$$

The corresponding ROC expression is given by

$$
P_{D}=Q\left[A Q^{-1}\left(P_{F A}\right)-B\right] \text {, }
$$

with

$$
\begin{aligned}
A & =\sqrt{\frac{K \epsilon_{s}+\epsilon_{x}}{K \epsilon_{s}+\epsilon_{\tilde{x}}}} \\
B & =\frac{\left(\epsilon_{x \tilde{x}}-\epsilon_{x}\right)}{\sqrt{\sigma^{2}\left(\epsilon_{\tilde{x}}+2 K \epsilon_{s}\right)}} .
\end{aligned}
$$

\section{THE UNDERWATER ACOUSTIC BARRIERS'07 (UAB07) EXPERIMENT SETUP}

The selected area for the UAB07 experiment was the Hopavågen Bay, in Sletvik (figure 1) approximately $100 \mathrm{~km}$ West from Trondheim (Norway). The experiment took place from 11 to 14 September 2007. The ground truth was relatively sparse and limited to archival data and some thermistor chain measurements. Bottom knowledge was limited to historical information.

\section{A. Environmental data}

Archival data of temperature and salinity profiles for the Hopavågen bay are shown in figure 2(a). These are mean profiles obtained during the month of September in the years from 1996 to 2006 (courtesy of Alexandra Neyts, NTNU). Figure 2(b) shows the temperature profile measured during the experiment with the thermistor string colocated with the vertical ROA. During this experiment the ROA was implemented with an Acoustic Oceanographic Buoy (AOB) [3]. The AOB was fitted with a low precision digital array of 16 temperature sensors. The array structure and details are described in [4]. In 


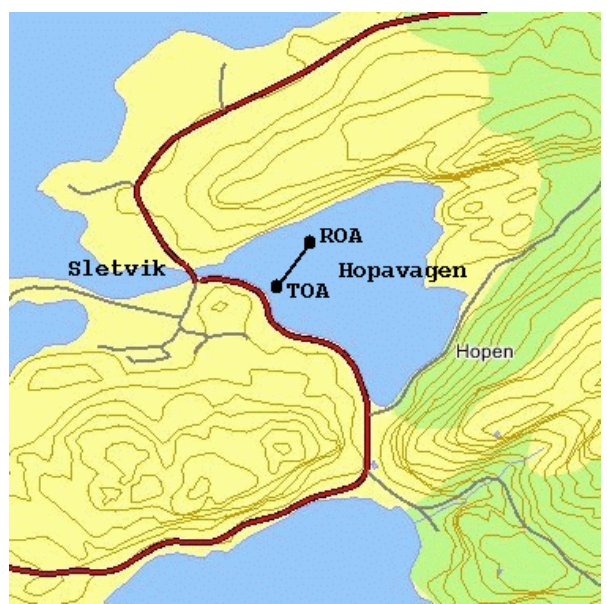

Fig. 1. UAB07 experiment area in the Hopavågen Bay: Transmitt Only Array (TOA) and Receive Only Array (ROA) approximate locations and transmission path (thick line).

short this is a series of $0.5^{\circ} \mathrm{C}$ precision sensors sampled at 4 $\mathrm{s}$ with 12-bit resolution. The two results show to be consistent.

(a)
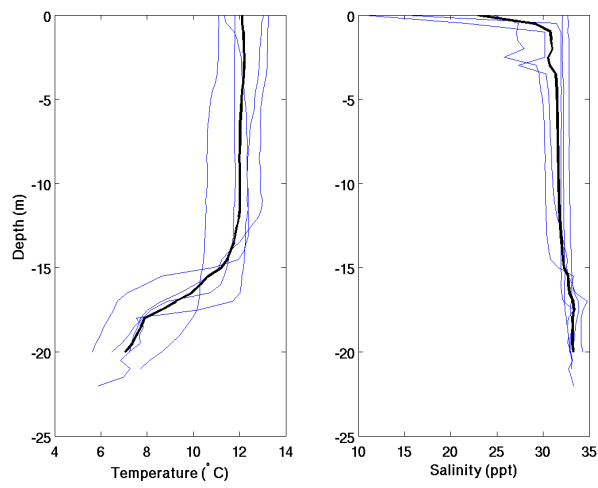

(b)

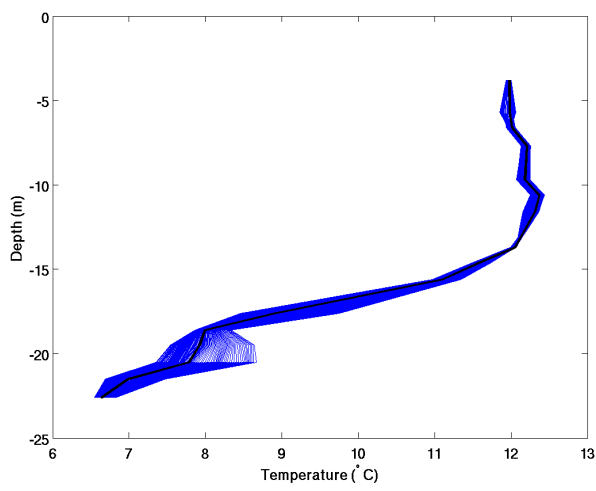

Fig. 2. Hopavågen bay mean temperature and salinity profiles from archival data for the month of September of the last 10 years (1996 - 2006) (a) and UAB07 measured temperature profile on day September 11 (b) (thick line is the mean temperature profile).

The bottom of the Hopavågen bay is traditionally described as being made of rock sand in most of the area that has a depth between 10 and $20 \mathrm{~m}$. In the deepest part of the bay, of about $30 \mathrm{~m}$ depth, the bottom is more muddy, with some organic marine sediments. There, the bottom may be anoxic during part of the summer. A complementary information about that area can be obtained from Bates [5], that performed a complete survey of the area using an echosounder system coupled with an additional processing for extracting bottom type information. The results show a variable bottom structure around the bay with a prodominance of fine sand to mud / silt in the deeper areas where acoustic signals were transmitted. This information was checked against ground truth coring. Bates also provided one of the rare bathymetry survey made of this Bay (figure 3) that shows a variable bathymetry from 0 to $30 \mathrm{~m}$, where the deepest part is a small area in the direction of the bay entrance in the left corner of the figure. Unfortunately up to now it has been impossible to obtain a coordinate transfer system so as to be able to position the UAB07 acoustic equipment in the map using global GPS coordinates.

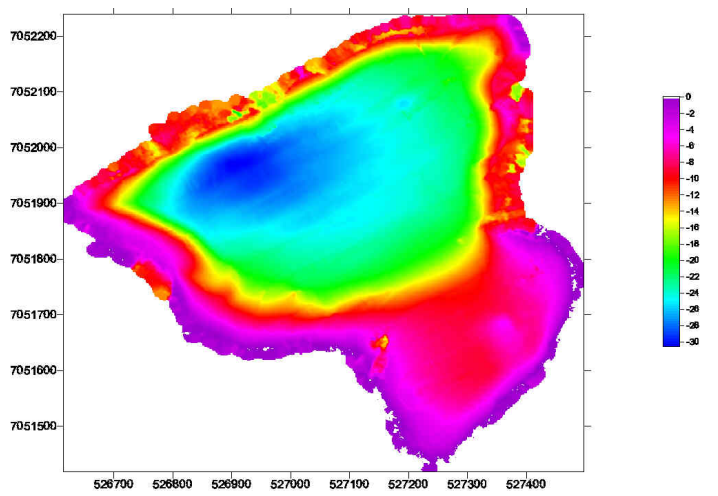

Fig. 3. Bathymetry recording of the Hopavågen bay (courtesy of NTNU / Bates [5]).

\section{B. Acoustic setup and experiment geometry}

The acoustic TOA was formed by two 916C Lubell acoustic sources arranged as shown in figure 4 . The top buoy position was measured with an hand held GPS and gave the following position: $63^{\circ} 35.5862^{\prime} \mathrm{N}, 009^{\circ} 32.4035^{\prime} \mathrm{E}$.

The AOB was attached to an horizontal line stretched between two existing moorings in the bay. The 16-hydrophone array was hand folded before deployment so as to comply with the reduced water depth at the array location resulting in an irregular hydrophone spacing between 3.8 and $22.6 \mathrm{~m}$ depth. A midlle array attach point was appropriately made so as to allow fixing the $8 \mathrm{Kg}$ weight in order to maintain the array as much vertical as possible.

Water depth at the array location was estimated to be between 26 and $28 \mathrm{~m}$. The AOB was slightly moving within an estimated radius of a few meters. This radius is extremely difficult to measure since its value is induced by a small movement that is within GPS accuracy. Using the TOA and 


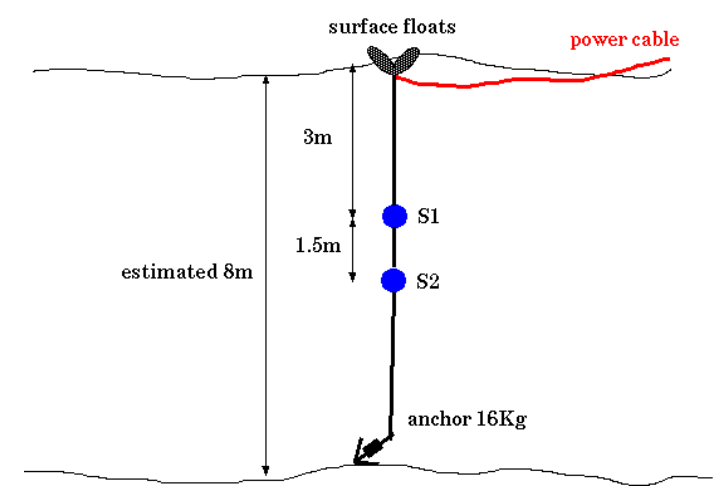

Fig. 4. sketch of the source array mooring and configuration.

the AOB GPS position allowed to estimate the TOA - ROA range to be $97 \mathrm{~m} \pm 4 \mathrm{~m}$.

\section{RESUlts}

\section{A. Propagation and modelling}

In order to obtain a perception of the expected detector performance a numerical simulation was carried out using a ray trace model in a realistic UAB'07 environment. The simulation requires a forward model being able to determine the acoustic field received at a certain location in time and space due to the superposition of the source array field and the scattering from the target object. So, the environment will be assumed as range dependent in bathymetry. The target object will be characterized by its size, position and density. All the energy will be assumed to propagate in the source - receiver plane. The numerical tool used was the TRACE/TRACEO pair [6], where TRACE is a standard Gaussian beam range dependent ray tracing package and TRACEO allows for defining an interfering object located in a pre-defined position in between the source and the receiver. Figure 5 is a sketch of the UAB07

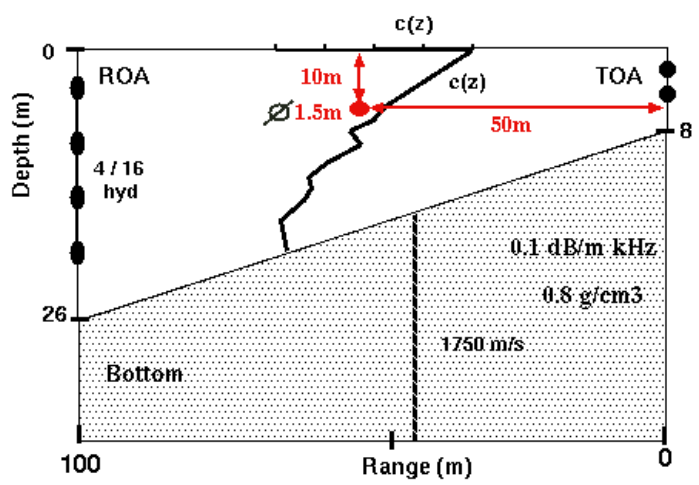

Fig. 5. UAB 07 derived scenario for simulations: the bathymetry was approximated by a five segment broken line between the source and the receiver.

scenario used in the simulations. The bottom was characterized by typical sand acoustic parameters and the bathymetry was aproximated by a five segment broken line between 0 and 100 $\mathrm{m}$ range. Figure 6 shows the simulated transmission loss field obained for a frequency band between 3.5 and $6.5 \mathrm{kHz}$ and a source depth of $3 \mathrm{~m}$, for the target free case in (a) and for the $1.5 \mathrm{~m}$ diameter cylinder target with a high reflectivity (c $=2000 \mathrm{~m} / \mathrm{s}$ ) located at $10 \mathrm{~m}$ depth and $50 \mathrm{~m}$ range in (b). It can be observed that from the transmission loss point of view, the field perturbation is minimum in this band and for this target size and position. In fact one of the questions that may arise is the possible variation of probability of detection with target type, size and position, that will be analyzed in the sequel. The perturbation produced by this target can be seen in

(a)

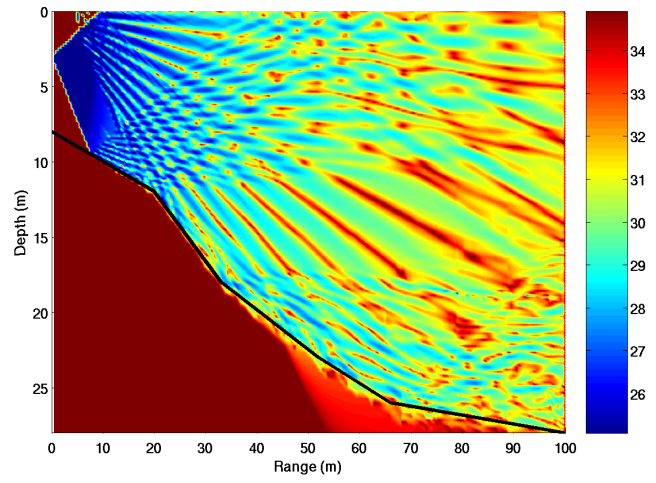

(b)

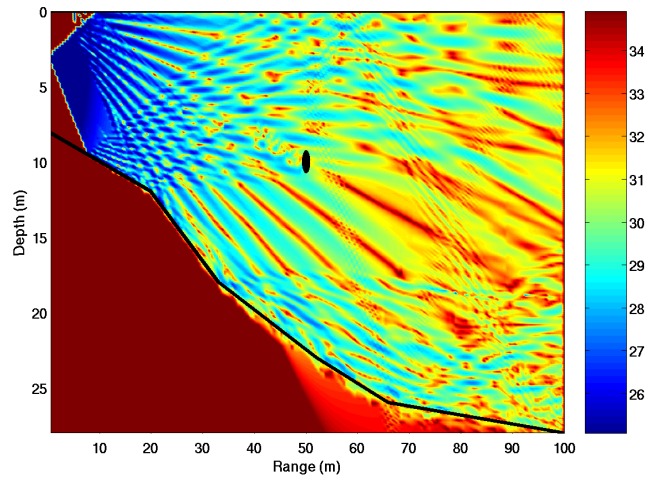

Fig. 6. Transmission loss field across the barrier line in the band [3.5,6.5] $k H z$, as given by TRACE (a) and by TRACEO with a target object of 1.5 $m$ diameter located at mid source - receiver range and mid water depth (black ellipse) (b). The thick black broken line represents the range dependent bathymtry used for the simulation.

terms of arrival structure in figure 7 for the target free case (a) and target perturbed case (b), with same source depth a target characteristics as in the previous transmission loss figure. These channel responses where computed at four discrete depths corresponding to those of the working hydrophones of the AOB (termed as canonical depths hereafter), in a 3 $\mathrm{kHz}$ band centered at $5 \mathrm{kHz}$. The perturbation induced by the target can be seen as a couple of arrivals on the first three hydrophones. Much of the effect of the target will depend on the amplitude of those arrivals which, due to ray tracing modeling limitations, can only be approximate. 
(a)

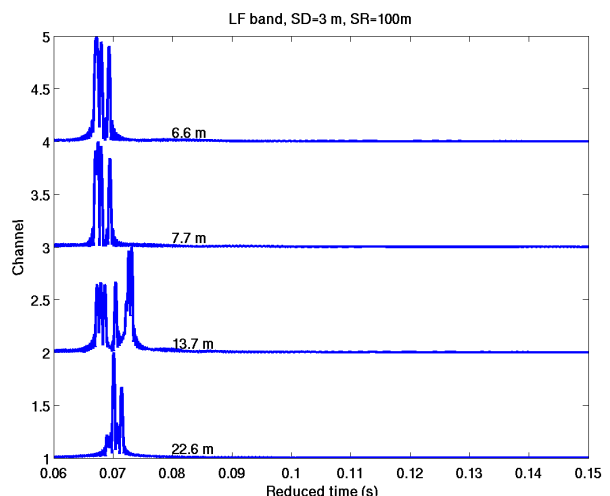

(b)

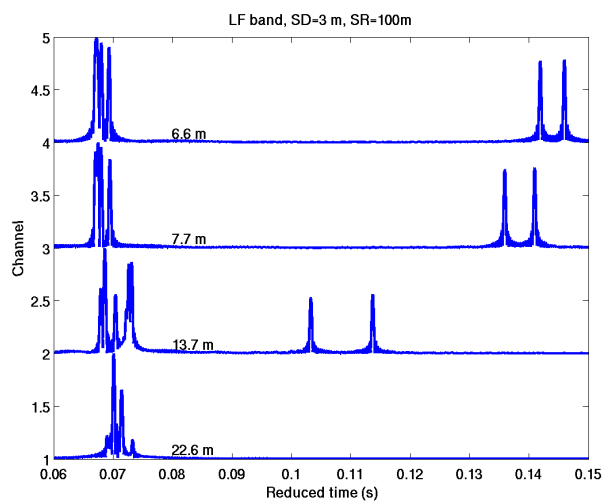

Fig. 7. Arrival patterns between a source at $3 \mathrm{~m}$ depth and four receivers located at marked depths, for a $3 \mathrm{kHz}$ bandwidth LFM signal centered in 5 $\mathrm{kHz}$ with no target present (a) and with a cylindrical $1.5 \mathrm{~m}$ diameter target at $10 \mathrm{~m}$ depth and mid source -receiver range $(b)$.

\section{B. Target detection performance}

Using both the detector expressions and the propagation tools of sections, II and IV-A, respectively, target detection performance can be computed for the various practical cases encountered in the UAB'07 scenario.

1) Optimal detector performance: The optimal detector ROC (9) allows us to obtain an upper bound for the system performance. The result for the UAB07 environment with 4 hydrophones at canonical depths operating in the $5 \mathrm{kHz}$ band to detect a $1.5 \mathrm{~m}$ diameter cylinder at mid water depth and range, is shown in figure 8 . Even with a small number of receivers, increasing the number of sources from 1 to 4 , provides a theoretical gain of up to $2 \mathrm{~dB}$. Moving the target over the range - depth plane allows us to determine the spatial variation of the probability of detection for a given probability of false alarm and SNR. The result is shown in figure 9 for one source at $3 \mathrm{~m}$ depth, $P_{f a}=10^{-3}$ and a SNR=15 dB. However, with an increase of 4 to 16 receivers evenly distributed in the water column will positively boost the system performance as shown in the ROC curve of figure 10. The spatial detection coverage is consequently improved with the addition of more receivers as shown in figure 11.

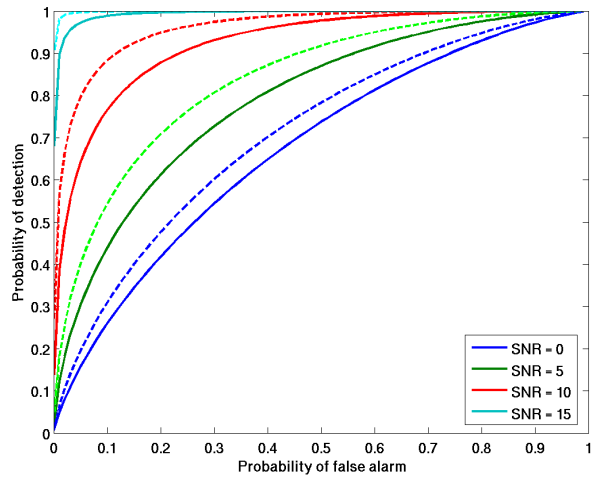

Fig. 8. Probability of detection of a $1.5 \mathrm{~m}$ diammeter rigid cylinder at mid source - receiver range, in the band [3.5,6.5] kHz as a function of probability of false alarm for various SNR: one source at $3 \mathrm{~m}$ depth (continous line) and 4 sources at 3, 4.5, 6 and $7 \mathrm{~m}$ depth (dashed lines) and four receivers at canonical depths.

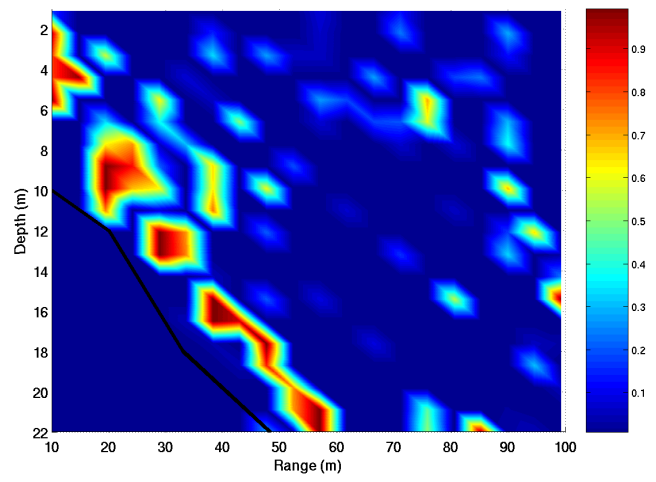

Fig. 9. Probability of detection of a $1.5 \mathrm{~m}$ diammeter rigid cylinder as a function of range and depth in the band $[3 / 5,6.5] \mathrm{kHz}$ for a $P_{f a}=10^{-3}$, $S N R=15 d B$, one source at $3 \mathrm{~m}$ depth and 4 sensors at canonical depths.

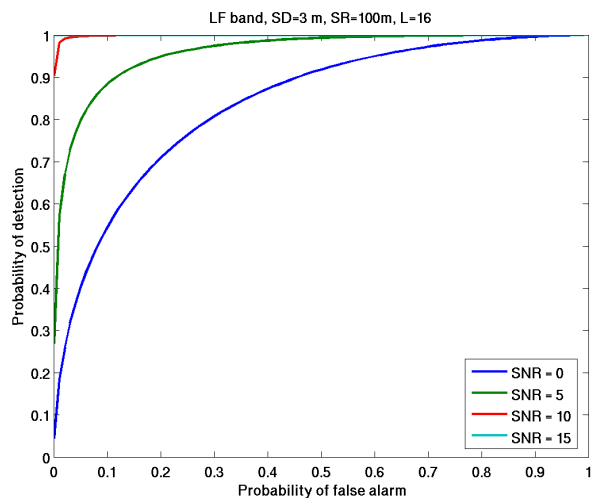

Fig. 10. Probability of detection of a $1.5 \mathrm{~m}$ diammeter rigid cylinder at mid source - receiver as a function of probability of false alarm for various SNR: one source at $3 \mathrm{~m}$ depth and 16 evenly distributed receivers.

2) suboptimal detectors' performance: As explained above, the implementation of the optimal detector is impossible since it assumes the perfect knowledge of the received signal both for the unmodified and modified cases, which is not available 


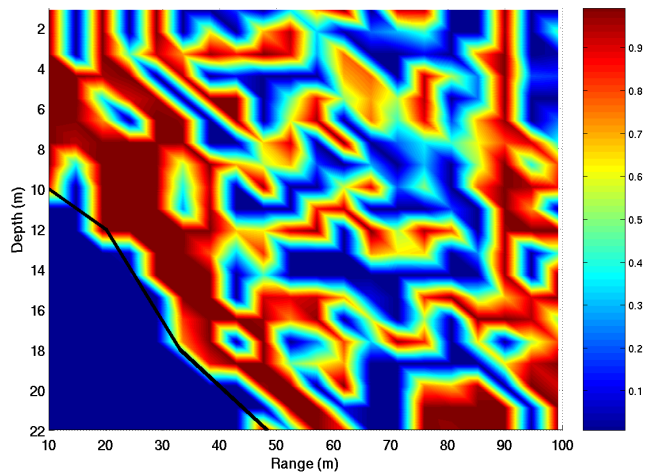

Fig. 11. Probability of detection of a $1.5 \mathrm{~m}$ diammeter rigid cylinder as a function of range and depth for a $P_{f a}=10^{-3}, S N R=15 \mathrm{~dB}$, four sources at 3, 4.5, 6 and $7 m$ depth and 16 sensors at canonical depths.

in practice. Three alternatives were suggested ranging from the trivial power based ping to ping detector, the non-channel based detector and the time-reversal (TR) double ping based detector, which expressions and underlying procedure was described in section II-B. Here these three detectors will be tested and compared under the UAB' 07 experimental conditions. The scenario was setup with 1 source at $3 \mathrm{~m}$ depth, 4 receivers at canonical depths and at $100 \mathrm{~m}$ range, the target has a circular cross-section object of $1.5 \mathrm{~m}$ diameter and crosses the barrier at $10 \mathrm{~m}$ depth and $50 \mathrm{~m}$ range. The results are shown in figure 12 for various SNR (color coded) and the three suboptimal implementations. The theoretical bound is also represented (thick lines).

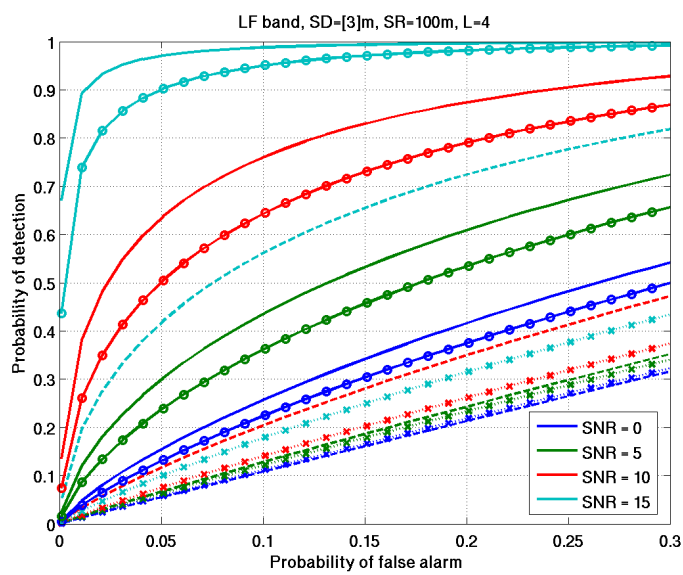

Fig. 12. Probability of detection of a $1.5 \mathrm{~m}$ diammeter rigid cylinder as a function of probability of false alarm for $S N R=[0,5,10,15] d B, 1$ source at $3 \mathrm{~m}$ depth and 4 receivers for: optimum case (continous), trivial powerbased detector (dashed), non-channel based ( $x$ dotted) and TR-based (circlescontinuos).

\section{Conclusions}

Port protection has been an intensive field of interest in the last few years. Alternative to or complimentary with other techniques, acoustic based detectors may offer viable means for covert yet powerfull systems. This paper presents a series of detectors using a standard setup of a transmit only array (TOA) and a receive only array (ROA), where the target is detected in a multi-static mode by forward scattering or standing field perturbation. Among the presented detectors this setup allows for a time-reversal (TR) based detector whose implementation is relatively simple and uses the actual acoustic channel to account for the matched-field receiver provided that the channel is stationnary and that a previous training phase can be carried out with no target present.

The results show that the non-channel based detector provides the lower performance, the trivial detector achieves a modest slightly better performance while the TR-based closely achieves the theoretical optimal performance. Although the system can be significantely improved, in particular by using more populated source and receiving arrays, it is believed that these results can be reproduced at sea in harbor like conditions.

\section{ACKNOWLEDGMENT}

The authors would like to thank funding from program POCI of FCT (Portugal) under project POCI/MAR/59008/2004 and from EU large infrastructure program (Hydralab III) during the UAB07 experiment. The support from Alexandra Neyts and Jens Hovem from NTNU, Trondheim (Norway) is greatly appreciated.

\section{REFERENCES}

[1] S.M. Kay", "Fundamentals of Statistical Signal Processing: Detection Theory", Prentice-Hall, Volume 2, New Jersey, USA, 1998.

[2] S.M. Jesus", "Active acoustic time-reversal for underwater acoustic barriers", 153th Meeting of the Acoustical Society of America, p.3204, vol. 121 - 5 Pt.2, Salt Lake City, USA, May 2007.

[3] A. Silva, F. Zabel and C. Martins, "Acoustic Oceanographic Buoy: a telemetry system that meets rapid environmental assessment requirements", Sea-Technology, Vol. 47, No. 9, pp. 15-20, September 2006.

[4] F. Zabel, C. Martins and A. Silva, "Analog 16-hydrophone vertical line array for the Acoustic - Oceanographic Buoy - AOB", Rep. 03/06, SiPLAB Report, University of Algarve, August 2006.

[5] C.R. Bates and E.J. Whitehead, "ECHOplus Measurements in Hopavågen Bay, Norway", Sea-Technology, vol. 42, no.6, pp. 34-43, June, 2001.

[6] O.C. Rodríguez, "The TRACE \& TRACEO ray tracing programs", SiPLAB Web page: www.siplab.fct.ualg.pt/models.shtml, 2008 